\section{Cardiac Arrest Survivors Attended by Out-of-Hospital Emergency Teams}

F. J. Mellado; J. J. Garcia del Aguila; F. Rosell; J. Colomina;

E. Olmeo

Public Utility for Health Emergencies, Spain

Objective: This study describes the clinical and epidemiological characteristics of patients with a cardiac arrest diagnosis attended by out-of-hospital emergency teams and later discharged from the hospital in Almeria (Andalusia, Spain), over a one-year period, as well as a one-month follow-up regarding quality of life.

Methods: All consecutive cases of cardiac arrest from 01 January-31 December 2003, in which emergency medical services (EMS) responded and attempted resuscitation, were reported and followed until discharged from the hospital, and later were followed-up after one month to check the quality of life of these patients.

Results: The out-of-hospital emergency teams attended to 96 patients with cardiac arrest. Of these, 23 patients (24\%) were admitted to the hospital alive and $73(76 \%)$ died in an out-of-hospital setting. Nine patients $(9.3 \%)$ were discharged from the hospital: eight males (89\%) and one female $(11 \%)$. The average age of the patients was 61 years old. The initial rhythms included five with electromechanical dissociation (56\%), two with asystole (22\%), and two with ventricular fibrillation (22\%). The out-of-hospital crew witnessed four of the cardiac arrests. Advanced life support (ALS) was performed in one case (11\%). The average of the time interval between cardiac arrest and the initiation of ALS was 3 minutes, 30 seconds. Six patients (67\%) had cardiac etiology. Three patients (33\%) had noncardiac etiology. In the one-month follow-up, all nine patients still were alive (100\%). Eight patients (89\%) reported good quality of life, and one patient $(11 \%)$ had bad quality of life (coma).

Conclusion: Survival rates reported were similar to other studies, although the number of patients is limited. These survival rates can be explained in part by the short time intervals between calls being received by the emergency dispatch center to the arrival of EMS on the scene. Cardiac etiology was predominant. Half of cardiac arrests were crew witnessed. For survivors, the quality of life was good. Keywords: cardiac arrest; emergency medical services (EMS); outof-hospital; quality of life; Spain; survival

Prebosp Disast Med 2005;20(2):s15

\section{Prehospital Use of Automated External Defibrillators (AEDs) in the First Three Minutes after Cardiac Arrest Due to Ventricular Fibrillation (VF) \\ S. Gromer; ${ }^{1}$ T. Kees, ${ }^{2}$ S. Steiger;, ${ }^{3}$ B. Domres, ${ }^{2}$ G. Klein; ${ }^{1}$ A. Königsrainer ${ }^{2}$ \\ 1. Robert-Bosch-Medical Centre, Germany \\ 2. Department of Surgery, University Hospital, Tuebingen, Germany \\ 3. Bjoern Steiger Foundation, Germany}

Introduction: Cardiac arrest is the most frequent cause of death in western populations. Each year in Germany, 130,000 patients die of a potentially treatable, cardiac arrest.
The use of automated external defibrillators (AEDs) currently is being evaluated worldwide.

This study evaluates the prehospital use of AEDs in the first three minutes after cardiac arrest due to ventricular fibrillation (VF).

Methods: The AEDs were used by a representative cross section of the German population including nurses, paramedics and emergency physicians, but mainly by medical laymen. Data was collected from 3,093 AEDs during an 18-month period.

Results: During this time, a total of 493 defibrillators were used in the first three minutes after cardiac arrest due to ventricular fibrillation. In 165 (33\%) cases, a successful defibrillation was performed, resulting in the patient's long-term survival.

Conclusion: The results of this study reveal the importance of educating medical laymen in the use of AEDs because quick therapy is an important factor in the successful treatment of VF. The analysis confirmes that AEDs can provide effective therapy in cardiac arrest, due to $\mathrm{VF}$, and can improve the outcome of cardiac arrest patients.

Keywords: automated external defibrillator (AED); cardiac arrest; prehospital care; ventricular fibrillation (VF) Prehosp Disast Med 2005;20(2):s15

Identification of Predictors for Survival in Out-ofHospital, Cardiac Arrest-Analysis of Prehospital Resuscitations in the Rescue Service of Dachau over Three Consecutive Years

K. Wilhelm; F. William; C. Günzel; H. Estner;

B. Rupprecht; D. Blaumeiser; $M$. Kaufer

Förderverein Rettungsdienst Dachauer Land e.V., Germany

Introduction: Sudden cardiac arrest is one of the most common causes of death in industrial countries. The main objective of this study was to identify possible predictors of survival in out-of-hospital, cardiac arrests, in order to improve training and organization of prehospital resuscitations.

Methods: The study evaluated all prehospital resuscitations in the district of Dachau over three consecutive years prospectively. Endpoints were death versus the return of spontaneous circulation (ROSC) on arrival at the emergency room (ER) and 30-day survival. All prehospital resuscitations were included, regardless of the cause (cardiac, trauma, other). The resuscitations were recorded via a standardized form completed by the paramedics after the incident.

Results: Between 2000 and 2003, a total of 269 resuscitations were recorded by the Dachau emergency medical service (EMS). Of these, 39.4\% ( $n=106)$ obtained the ROSC and admitted to the ER. A total of $49(18.2 \%)$ patients survived the first 24 hours and $26(9.7 \%)$ patients were still alive after 30 days, resulting in a 30 -day mortality of $90.3 \%$.

Significant differences in survival could be demonstrated in patients with ventricular fibrillation (VF) $(n=82)$ versus asystole, electro-mechanical dissociation (EMD), or other rhythms ( $\mathrm{n}=187$ ) as the primary electrocardiogram (ECG) (30-day survival $20.7 \%$ vs. $4.8 \%$ ). Also, in the patients with witnessed cardiac arrest ( $n=163$ vs. $n=106$ non-witnessed), a significant difference in 30-day survival rate $(14.1 \%$ vs. $2.8 \%)$ was found. 